\title{
Article \\ The Role of Polyphosphate in Motility, Adhesion, and Biofilm Formation in Sulfolobales
}

\author{
Alejandra Recalde ${ }^{1,2} \mathbb{D}$, Marleen van Wolferen ${ }^{2} \mathbb{D}$, Shamphavi Sivabalasarma ${ }^{2} \mathbb{D}$, Sonja-Verena Albers ${ }^{2}$, \\ Claudio A. Navarro ${ }^{1}$ and Carlos A. Jerez ${ }^{1, *}$ \\ 1 Laboratory of Molecular Microbiology and Biotechnology, Department of Biology, Faculty of Sciences, \\ University of Chile, Santiago 8320000, Chile; alejandra.recalde@biologie.uni-freiburg.de (A.R.); \\ clnavarrol@gmail.com (C.A.N.) \\ 2 Laboratory of Molecular Biology of Archaea, Institute of Biology II-Microbiology, University of Freiburg, \\ 79085 Freiburg, Germany; marleen.van.wolferen@biologie.uni-freiburg.de (M.v.W.); \\ shamphavi.sivabalasarma@biologie.uni-freiburg.de (S.S.); sonja.albers@biologie.uni-freiburg.de (S.-V.A.) \\ * Correspondence: cjerez@uchile.cl
}

Citation: Recalde, A.; van Wolferen, M.; Sivabalasarma, S.; Albers, S.-V.; Navarro, C.A.; Jerez, C.A. The Role of Polyphosphate in Motility, Adhesion, and Biofilm Formation in Sulfolobales. Microorganisms 2021, 9, 193. https:// doi.org/10.3390/microorganisms9010193

Received: 6 November 2020 Accepted: 19 December 2020 Published: 18 January 2021

Publisher's Note: MDPI stays neutral with regard to jurisdictional clai$\mathrm{ms}$ in published maps and institutional affiliations.

Copyright: $\odot 2021$ by the authors. Licensee MDPI, Basel, Switzerland. This article is an open access article distributed under the terms and conditions of the Creative Commons Attribution (CC BY) license (https:// creativecommons.org/licenses/by/ $4.0 /)$.

\begin{abstract}
Polyphosphates (polyP) are polymers of orthophosphate residues linked by high-energy phosphoanhydride bonds that are important in all domains of life and function in many different processes, including biofilm development. To study the effect of polyP in archaeal biofilm formation, our previously described Sa. solfataricus polyP (-) strain and a new polyP (-) S. acidocaldarius strain generated in this report were used. These two strains lack the polymer due to the overexpression of their respective exopolyphosphatase gene ( $p p x)$. Both strains showed a reduction in biofilm formation, decreased motility on semi-solid plates and a diminished adherence to glass surfaces as seen by DAPI ( $4^{\prime}, 6$-diamidino-2-phenylindole) staining using fluorescence microscopy. Even though $\operatorname{arlB}$ (encoding the archaellum subunit) was highly upregulated in S. acidocardarius polyP $(-)$, no archaellated cells were observed. These results suggest that polyP might be involved in the regulation of the expression of archaellum components and their assembly, possibly by affecting energy availability, phosphorylation or other phenomena. This is the first evidence indicating polyP affects biofilm formation and other related processes in archaea.
\end{abstract}

Keywords: sulfolobales; Saccharolobus solfataricus; Sulfolobus acidocaldarius; inorganic polyphosphates; biofilm; archaellum

\section{Introduction}

Polyphosphates (polyP) are polymers of inorganic orthophosphate that can reach hundreds to thousands of residues in size. PPK and PPX are the two main enzymes related to polyP metabolism in Prokaryotes. PPK synthesizes polyP, consuming ATP as the polyP chain grows, generating ADP and polyP $(n+1)$ as final products. On the other hand, PPX degrades polyP starting from its last phosphate group, releasing Pi and polyP $(n-1)$ in the process [1]. In archaea, PPX has been identified either by experimental [2] or bioinformatic analysis [3]. Nevertheless, even though polyP accumulates in some Crenarchaeotes, no archaeal PPK homolog has been found so far, suggesting a different enzyme involved in polyP synthesis in this phylum.

Many functions of polyP have been revealed in bacteria such as: ATP substitution, helping in nutrition starvation, functioning in metal resistance, and several other roles [1,4]. Some of those functions are related to biofilm formation, motility, and sporulation [5-8] as shown with several PPK and/or PPX mutants in diverse bacterial species.

A ppk deletion mutant in Pseudomonas aeruginosa showed a thinner and more uniform biofilm when compared to the wild type (WT) strain and lacked cells forming clusters along with water channels [6]. This mutant was defective in swimming, swarming, twitching and adhesion to surfaces. All of these phenomena are related to biofilm formation. Involvement 
of polyP in biofilm development, sporulation and even virulence was also shown in Bacillus cereus [7] and Campylobacter jejuni [5].

In E. coli, the role of polyP in biofilm generation has been studied in more detail. Rather than the intracellular level of the polymer, polyP degradation triggers biofilm formation in stationary phase via Type 2 Autoinducers (AI-2), specifically the LuxS Quorum Sensing system [9]. A correlation between polyP levels and AI-1 and AI-2 was also seen in P. aeruginosa [6].

The possible role of polyP in biofilm formation in archaea has not been explored. Archaea lack AI-2, suggesting that regulation in these microorganisms functions differently. Regulation of biofilm formation in Sulfolobus acidocaldarius involves two transcriptional regulators belonging to the Lrs-14 family: encoded by Saci_1223 and Saci_0446, the latter is known as AbfR1 (Archaeal Biofilm Regulator 1) [10]. Saci_1223 is a positive regulator, inducing biofilm formation, whereas AbfR1 is a negative regulator that inhibits biofilm development by repressing extracellular polymeric substances (EPS) synthesis and promoting swimming motility by using archaella. AbfR1 is regulated by phosphorylation [11] and cannot impair biofilm formation when phosphorylated. The pathway for EPS synthesis has not been described yet for Sulfolobales.

Here we show that polyP is also related to biofilm formation in archaea, affecting both attachment of cells to surfaces and motility, apparently through assembly of the archaellum (archaeal motility structure), in two model crenarchaeotes Sa. solfataricus and S. acidocaldarius.

\section{Materials and Methods}

\subsection{Strains and Growth Conditions}

All strains used in this study are cited in Supplementary Table S1.

Sa. solfataricus M16 and its polyP (-) strain [12], as well as S. acidocaldarius MW001 and its corresponding polyP (-) strain were grown as before at $75^{\circ} \mathrm{C}$ by shaking at $150 \mathrm{rpm}$ in Brock Medium [12], $\mathrm{pH} 3$ and supplemented with $0.1 \%(p / v) \mathrm{N}-\mathrm{Z}$ amine (Sigma-Aldrich ${ }^{\circledR}$, Merk KGaA, Burlington, MA, USA), $0.2 \%(p / v)$ glucose and $0.01 \mathrm{mg} / \mathrm{mL}$ uracil only in the case of uracil autotrophs. For overexpression of PPX (described below), $0.2 \%(w / v)$ D-arabinose (Sigma-Aldrich ${ }^{\circledR}$, Merk KGaA, Burlington, MA, USA) was added.

\subsection{Construction of a S. acidocaldarius Mutant Lacking PolyP}

To generate recombinant S. acidocaldarius, the ppx gene (Saci_2018) was amplified from S. acidocaldarius MW001 genomic DNA using PCR with primers 10904 and 10905 (Table S2). The obtained product was cloned into pSVAaraFX-H6 [13] using SapI (New England Biolabs., Ipswich, MA, USA), resulting in pSVA12801. The plasmid was sequenced.

Before being introduced in S. acidocaldarius MW001, the plasmid was methylated by transformation and purification from E. coli ER1821 strain. Methylated plasmids were transformed to competent S. acidocaldarius MW001 as described in [14] and plated on Brock first selection plates. Colonies were picked and grown in Brock medium supplemented with $\mathrm{N}-\mathrm{Z}$ amine and Glucose.

The overexpression of PPX enzyme was induced for $3 \mathrm{~h}$ with $0.2 \%$ D-arabinose while growing in liquid Brock medium with supplements. Cells were collected by centrifugation and boiled in protein buffer (0.5 M Tris- $\mathrm{HCl}$, pH 6.8, $65 \mathrm{mM}$ glycerol, 10\% SDS, 2\% 2mercapto ethanol, $0.025 \%$ bromophenol blue) at $100{ }^{\circ} \mathrm{C}$ for $5 \mathrm{~min}$.

\subsection{PolyP Extraction and Quantification}

Cultures of $S$. acidocaldarius were grown as described in Section 2.1. PolyP from S. acidocaldarius cells were isolated as previously described [12]. In brief, $5 \mathrm{~mL}$ culture was centrifuged for $15 \mathrm{~min}$ at $4500 \times \mathrm{g}$. Cell pellet was resuspended in prewarmed $300 \mu \mathrm{L}$ of $4 \mathrm{M}$ guanidine isothiocyanate (GITC), $50 \mathrm{mM}$ Tris- $\mathrm{HCl} \mathrm{pH} 7.0$ solution. The suspension was subjected to vortex and heated for $3 \mathrm{~min}$ at $95^{\circ} \mathrm{C}$. Twenty $\mu \mathrm{L}$ of each sample was saved for protein quantification using Coomassie Plus Protein Assay Reagent (PIERCE ${ }^{\mathrm{TM}}$, 
Thermo Fisher Scientific, Waltham, MA, USA). Thirty $\mu$ L of $10 \%$ SDS was added to each sample and then heated again at $95^{\circ} \mathrm{C}$ for $5 \mathrm{~min}$. To bind polyP, $5 \mu \mathrm{L}$ of silica (Glassmilk) and $300 \mu \mathrm{L}$ of ethanol (100\%) were added and mixed by vortexing followed by heating at $95{ }^{\circ} \mathrm{C}$ for $30 \mathrm{~s}$. After $1 \mathrm{~min}$ centrifugation at $13,000 \times g$, the supernatant was removed and the glassmilk pellet was resuspended in $200 \mu \mathrm{L}$ of ice cool New Wash Buffer $(5 \mathrm{mM}$ Tris- $\mathrm{HCl}$ pH 7.5, $50 \mathrm{mM} \mathrm{NaCl}, 5 \mathrm{mM}$ EDTA, 50\% ethanol). After centrifugation, glassmilk was resuspended in $100 \mu \mathrm{L}$ of a solution containing $50 \mathrm{mM}$ Tris- $\mathrm{HCl}$ pH 7.0, $5 \mathrm{mM} \mathrm{MgCl}$, $5 \mu \mathrm{g} / \mathrm{mL}$ DNase and $5 \mu \mathrm{g} / \mathrm{mL}$ RNase and incubated for $30 \mathrm{~min}$ at $37^{\circ} \mathrm{C}$ to eliminate nucleic acids. The pellet was washed twice with New Wash Buffer. Finally, a total of $100 \mu \mathrm{L}$ of polyP-containing solution was obtained and polyP was recovered from the glassmilk after repeatedly vortexing with $50 \mu \mathrm{L}$ of water, heating at $95^{\circ} \mathrm{C}$ and centrifugation. If not measured immediately, samples were frozen and stored at $-20{ }^{\circ} \mathrm{C}$.

Quantification of total $\mathrm{P}_{\mathrm{i}}$ was done with EnzChek Phosphate Assay kit (Invitrogen ${ }^{\mathrm{TM}}$, Thermo Fisher Scientific, Waltham, MA, USA) following manufacturer's instructions and expressed as nmol of $\mathrm{P}_{\mathrm{i}}$ per $\mathrm{mg}$ of protein. Thirty $\mu \mathrm{L}$ of polyP containing solution was subjected to acid hydrolysis with $30 \mu \mathrm{L} 2 \mathrm{~N} \mathrm{HCl}$ for $30 \mathrm{~min}$ at $95{ }^{\circ} \mathrm{C}$ to release inorganic phosphate $\left(\mathrm{P}_{\mathrm{i}}\right)$, followed by quantification.

\subsection{Microtitration Plates Assays}

Cells were grown to stationary phase in Brock medium ( $\mathrm{pH}$ 3.5) supplemented with $0.1 \% \mathrm{~N}$-Z-amine and $0.2 \% \mathrm{D}$-arabinose or uracil in 96 wells microtitration plates covered with gas permeable sealing membrane (Breathe-Easy, Diversified Biotech, Boston, MA, USA) at $75{ }^{\circ} \mathrm{C}$ for 2 or 3 days inside a humidity chamber to avoid evaporation. The starting $\mathrm{OD}_{600}$ was 0.03 for $S a$. solfataricus and 0.01 for $S$. acidocaldarius, as previously described by Koerdt et al. [15]. After 2 or 3 days, plates were cooled down, the supernatant was placed in a new plate and the $\mathrm{OD}_{600}$ was determined by an Epoch luminometer (BioTek instruments, Agilent Technologies, Inc., Santa Clara, USA). Ten $\mu \mathrm{L}$ of $0.5 \%$ crystal violet solution (CV) was added to each well containing the biofilm followed by incubation for $10 \mathrm{~min}$. Sessile cells were washed with Brock ( $\mathrm{pH} 5$ ) medium or water, and CV attached to the biofilm was released by using $200 \mu \mathrm{L}$ of $30 \%$ acetic acid. The CV released was determined by measuring the $\mathrm{OD}_{570}$. The $\mathrm{OD}_{570} / \mathrm{OD}_{600}$ correlation index was used to determine biofilm formation efficiency.

\subsection{Confocal Laser Microscopy}

For Confocal Laser Microscopy (CLM), cells were grown in $35 \mathrm{~mm}$ petri dishes ( $\mu$ dishes; Ibidi; Martinsried) [15]. Culture medium was exchanged every $24 \mathrm{~h}$, and after 3 days, the supernatant was exchanged for $2 \mathrm{~mL}$ Brock $(\mathrm{pH}$ ) and biofilm was stained with 3.6 $\mu \mathrm{L}$ of DAPI $\left(4^{\prime}\right.$,6-diamidino-2-phenylindole) $(500 \mu \mathrm{g} / \mathrm{mL}), 15 \mu \mathrm{L}$ ConA-fluoresceine (5 mg/mL) and IB4-Alexa568 (lectin IB4 from Griffonia simplicifolia, Invitrogen ${ }^{\mathrm{TM}}$, Thermo Fisher Scientific, Waltham, MA, USA) was used for CLM. Staining was done for $30 \mathrm{~min}$ in the dark at room temperature.

Biofilms were observed under microscope ZEISS Observer 1 with $63 \times$ objective

\subsection{Adhesion Assays}

For adhesion assays, $40 \mathrm{~mL}$ of $\mathrm{Sa}$. solfataricus or $\mathrm{S}$. acidocaldarius cultures with an initial $\mathrm{OD}_{600}$ of 0.03 and 0.01 , respectively, were grown to $\mathrm{OD}_{600}$ of $0.5-0.7$ for $24 \mathrm{~h}$ at $75^{\circ} \mathrm{C}$ and $150 \mathrm{rpm}$ in a $100 \mathrm{~mL}$ Schott flask with a glass slide inside. After that period, the glass slide was removed, washed twice with Brock medium ( $\mathrm{pH} 5)$, and cells attached were fixed with $4 \%$ formaldehyde dissolved in Brock medium ( $\mathrm{pH}$ ). Cells and EPS were stained with $6 \mu \mathrm{L}$ DAPI $(300 \mu \mathrm{g} / \mathrm{mL})$ and $15 \mu \mathrm{L}(5 \mathrm{mg} / \mathrm{mL})$ ConA dissolved in $1 \mathrm{~mL}$ Brock medium (pH 5) for $30 \mathrm{~min}$ in the dark. After staining, slides were washed twice with Brock medium and air dried. Cells in the back part of the slide were removed with $70 \%$ ethanol. Slides were observed by using the TIRF Observer 1 from ZEISS microscope with a $100 \times$ objective. Ten 
pictures from different fields were taken from each of 3 biological replicates and processed with Fiji (ImageJ) [16].

\subsection{Motility Assays}

Motility was analyzed on semi-solid gelrite plates. Motility plates were made with Brock medium pH 5, 0.15\% gelrite and supplemented with $0.0001 \% \mathrm{~N}-\mathrm{Z}$ amine, $0.2 \%$ D-arabinose and uracil when needed. Cells for inoculation were grown to an $\mathrm{OD}_{600}$ between 0.4 and 0.6 , placed as a spot on the surface of the motility plates and air dried. Plates were incubated for 4 days in the case of $S$. acidocaldarius strains, and 9 days for $\mathrm{Sa}$. solfataricus strains inside a humidity chamber. Finally, the swimming radius/area of each spot was measured.

\subsection{Total RNA Extraction and cDNA Synthesis}

To study the expression of genes of interest, cells were grown in large $150 \mathrm{~mm}$ petri dishes for biofilm formation during 3 days at $75{ }^{\circ} \mathrm{C}$ and no agitation inside a humidity chamber, exchanging the medium after the first $24 \mathrm{~h}$. Cells were grown in Brock medium, starting at an initial $\mathrm{OD}_{600}$ of 0.03 . For polyP (-) strains, D-arabinose was added. For planktonic cells, cultures were grown in the same conditions but in Erlenmeyer flasks.

After 3 days, the supernatant from the biofilms was removed and cells from the biofilm were washed twice with Brock medium and scraped from the bottom with a cell scraper and $20 \mathrm{~mL}$ of fresh medium. Biofilm and planktonic cells (10 mg wet weight) were harvested by centrifugation $(7700 \times g$ for $15 \mathrm{~min})$. Cell pellets were washed three times with Brock's medium and lysed as previously described [17]. RNA was extracted by using TRIzol (Invitrogen ${ }^{\mathrm{TM}}$, Thermo Fisher Scientific, Waltham, MA USA) as described by the manufacturer. Remaining DNA was eliminated by adding $40 \mathrm{U}$ of TURBO DNA-free DNase (Invitrogen ${ }^{\mathrm{TM}}$, Thermo Fisher Scientific, Waltham, MA, USA) following manufacturer's instructions. $0.8 \mu \mathrm{g}$ of total RNA was reverse transcribed for cDNA synthesis using ImProm-II (Promega, Madison, WI, USA), $0.5 \mu \mathrm{g}$ of random hexamers (Promega, Madison, WI, USA) and $3 \mathrm{mM} \mathrm{MgCl}{ }_{2}$ for $1 \mathrm{~h}$ at $42{ }^{\circ} \mathrm{C}$. Three biological replicates were used for every experimental condition.

\subsection{Primer Design and Real-Time RT-PCR}

Primers for qRT-PCR were designed using Primer3 Software and the annotated genome of Sa. solfataricus P2 and S. acidocaldarius DSM639, as they are the WT strains $[14,18]$ (Table S1). All primers are listed in Table S2. To check primer specific annealing and optimal melting temperature, PCR reactions were carried out with Taq DNA polymerase from Promega following manufacturer's instructions and the products were separated by gel electrophoresis ( $1 \%$ agarose).

Gene expression was analyzed with the 96-well PikoReal-Time PCR System (Thermo Fisher Scientific, Waltham, USA). Five $\mu$ L of KAPA SYBR ${ }^{\circledR}$ FAST $2 X$ (Sigma-Aldrich ${ }^{\circledR}$, Merk KGaA, Burlington, USA) was used along $0.2 \mu \mathrm{L}$ of each primer and $0.5 \mu \mathrm{L}$ of a 1:20 dilution of the cDNA.

The efficiency of each pair of primers was calculated from the average slope of a linear regression curve, constructed from qPCRs using a 10-fold dilution series (10 pg-10 ng) of Sa. solfataricus M16 or S. acidocaldarius MW001 chromosomal DNA as template. Cq values (quantification cycle) after 40 cycles were automatically determined by PikoReal Software 2.1 (Thermo Fisher Scientific, Waltham, MA, USA). Cq values of each transcript of interest was standardized to the Cq value of the 16s and/or 30s rRNA gene. At least 3 biological replicates of each assessed condition and 2 technical replicates per qPCR reaction were performed. Rps2P and $16 \mathrm{~S}$ rRNA were used as housekeeping genes.

\subsection{Starvation Induction Experiment}

S. acidocaldarius MW001 and polyP (-) were grown in Brock medium and supplemented with $0.1 \%(p / v) \mathrm{N}-\mathrm{Z}$ amine (Sigma-Aldrich ${ }^{\circledR}$, Merk KGaA, Burlington, USA), $0.2 \%$ 
$(p / v)$ dextrose and $0.01 \mathrm{mg} / \mathrm{mL}$ uracil only in the case of S. acidocaldarius MW001. For overexpression of PPX, 0.2\% (w/v) D-arabinose (Sigma-Aldrich ${ }^{\circledR}$, Merk KGaA, Burlington, USA) was added. A control of $S$. acidocaldarius MW001 with plasmid pSVA12801 without induction was used.

After reaching an OD600 of 0.4-0.5, cells were centrifuged for $10 \mathrm{~min}$ at $4400 \mathrm{rpm}$ and $70{ }^{\circ} \mathrm{C}$ and resuspended in starvation medium without supplements, but with uracil and D-arabinose when needed. Cells were grown for $4 \mathrm{~h}$ at $75^{\circ} \mathrm{C}$ and $150 \mathrm{rpm}$, and then used for Transmission Electron Microscopy.

\subsection{Transmission Electron Microscopy}

Five $\mu \mathrm{L}$ of cells from the starvation experiment were applied on a glow-discharged 300 mesh formvar and carbon-coated copper grid (Plano GmbH, Wetzlar, Germany) and incubated for $10 \mathrm{~s}$. The excess liquid was blotted away and the application step was repeated 8 times subsequently. Afterwards cells were stained with $2 \%(w / v)$ uranyl acetate. Imaging was performed using Zeiss Leo 912 Omega (Tungsten) (Carl Zeiss, Oberkochen, Germany) operated at 80 kV. Images were taken with Dual Speed 2K-On-Axis chargedcoupled device (CCD) camera TRS, Sharp-Eye (TRS Systems, Moorenweis, Germany).

\subsection{Statistical Analysis}

Data obtained was subjected to analysis of variance (ANOVA) and a Bonferroni's test in the Prism 8 GraphPad software.

\section{Results}

\subsection{Biofilm Formation}

Microtiter plates assays were performed to compare biofilm formation of background and polyP (-) strains of both Sa. solfataricus and S. acidocaldarius. Lack of polyP in S. acidocaldarius MW001 transformed with pSVA12801 was confirmed after $3 \mathrm{~h}$ post induction of $p p x$ with $0.2 \%$ D-Arabinose (Figure S1), as described before for Sa. solfataricus [12]. A control of cells with plasmid but no induction was use for some experiments and they will be referred as Sa. solfataricus M16-PPX and S. acidocaldarius MW001-PPX.

Sa. solfataricus biofilms of polyP (-) and background strains at days 2 and 3 of growth showed significant differences as indicated by the 570/600 nm index (Figure 1, $570 / 600$ ratio panel). The number of planktonic cells remained similar for all conditions (Figure 1, upper panels, Planktonic cells) as was previously described [12]. The differences were therefore mainly due to changes in biofilm formation as shown by crystal violet staining (biofilm) (Figure 1, Biofilm masses).

A similar but less pronounced effect could be observed for S. acidocaldarius (Figure 1, lower panels), with polyP (-) strain showing decreased levels of biofilm formation when compared to the background strain after 3 days of growth. Planktonic cells showed no significant differences. In addition, growth curves of both strains were similar (Figure S2). In both cases, cells with plasmid but no induction of ppx (M16-PPX and MW001-PPX) showed similar biofilm production compared with their background counterparts.

Here we have therefore shown that comparable to what is known from many bacteria [5-7], Sulfolobales polyP (-) strains also showed reduced biofilm formation. 


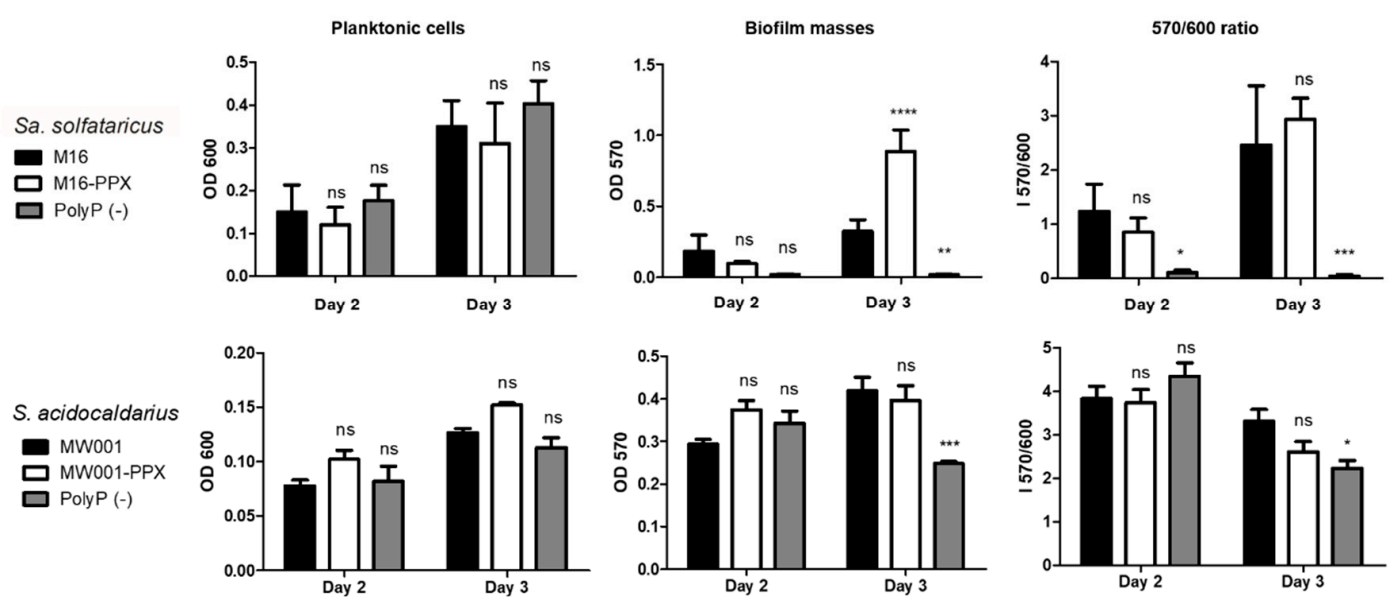

Figure 1. Effect of the lack of polyphosphates (polyP) in biofilm formation in Sa. solfataricus and S. acidocaldarius on days 2 and 3 of growth. Microtitrate assay of background strains (M16 and MW001), cells transformed with plasmid but no induction of $p p x$ (M16-PPX and MW001-PPX) and polyP (-) cells from each species. The biofilm mass corresponds to Cristal violet attached to the biofilm measured at $570 \mathrm{~nm}$. ANOVA test: ${ }^{* * *}$ indicating $p \leq 0.0001,{ }^{* *} p \leq 0.001,{ }^{* *} p \leq 0.01$, $* p \leq 0.05$ and ns: no significative.

\subsection{Confocal Laser Microscopy}

To characterize the biofilm morphology of polyP (-) strains, CLM was done on 3 days old biofilms stained with DAPI (DNA), ConA (staining $\alpha$-mannopyranosyl and $\alpha$-glucopyranosyl residues) and IB4 (for $\alpha$-D-galactosyl residues). The biofilm of Sa. solfataricus M16 showed a morphology similar to that previously described for Sa. solfataricus M16 strain (Figure 2) [15], with some cumulus of EPS and a uniform distribution of cells, but the biofilm was thinner in our strain (Figure 2B). As previously described, Sa. solfataricus M16 excretes EPS in low amounts [15] (Figure 2A, ConA+IB4 channels).

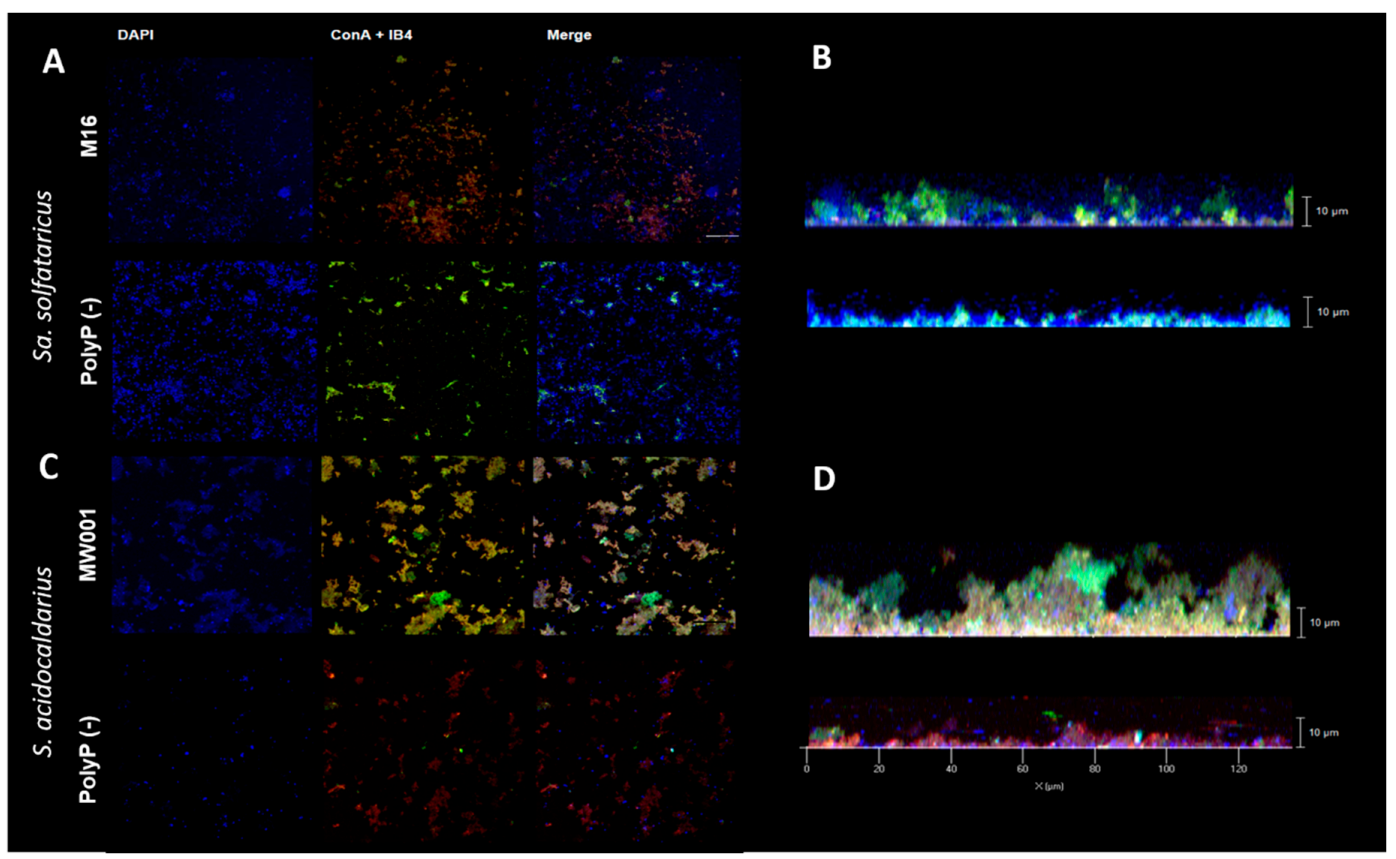

Figure 2. Confocal Laser Microscopy showing differences between wild type (WT) and polyP (-) strains biofilms in day 3. Biofilms were stained with DAPI (4',6-diamidino-2-phenylindole), ConA and IB4. Biofilms from (A) M16 and polyP (-) strains of Sa. solfataricus and (C) MW001 and polyP (-) of S. acidocaldarius in DAPI channel (blue), merge of ConA (green) and IB4 (red) channels (extracellular polymeric substances (EPS)) and merge of all three channels. (B,D) Z stack of WT and polyP (-) strains in both species, merge with all three channels. 
Sa. solfataricus polyP (-) biofilms were thinner than the background strain biofilms (6 versus $10 \mu \mathrm{m}$ ) and contained less cells and very low amounts of EPS (Figure 2A). Additionally, the composition of the glycans present in the biofilm seemed to be different when comparing both strains: biofilms of $S a$. solfataricus polyP (-) mainly contained $\alpha$-mannopyranosyl and $\alpha$-glucopyranosyl residues, (Figure 2A, green, ConA), whereas its background strain contained more $\alpha$-D-galactosyl residues (Figure 2A, red, IB4).

S. acidocaldarius polyP (-) also showed a thinner biofilm when compared to the background strain (Figure 2D). Moreover, lower amounts of $\alpha$-mannopyranosyl and $\alpha$ glucopyranosyl sugars could be observed (Figure 2C, green, ConA).

\subsection{Phenotypes Related to Surface Structures: Adhesion to Glass Surface and Motility Assays}

The archaellum in Sa. solfataricus and S. acidocaldarius plays an important role in attachment to surfaces $[19,20]$. In S. acidocaldarius there is an additional surface appendix that is involved in adhesion and biofilm initiation, called Adhesive Archaeal Pilus (Aappilus) [21].

To test if the surface attachment of polyP (-) strains was also impaired, all strains were grown in shaking cultures each with a glass slide inside. Both polyP $(-)$ strains showed reduced attachment to the glass surfaces when compared to their corresponding background strains (Figure 3). This difference in initial cell attachment could (partially) explain the observed reduced biofilm formation of the polyP (-) strains (Figure 1).

A

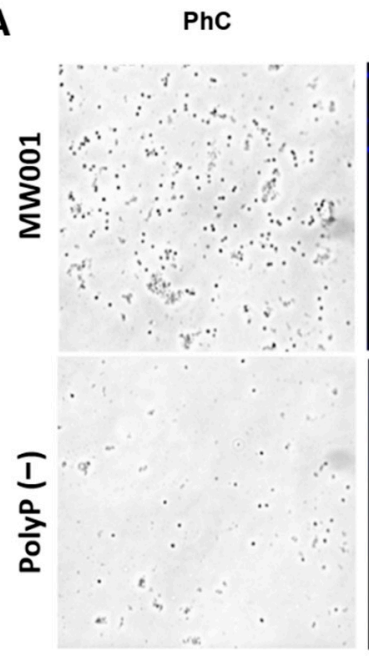

DAPI

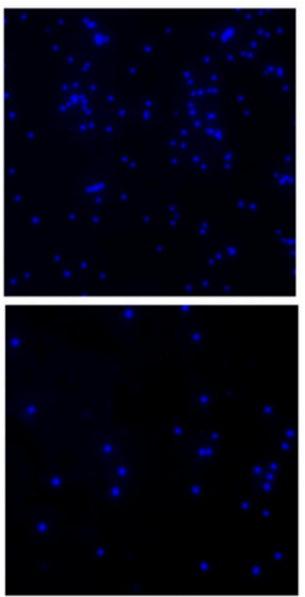

B

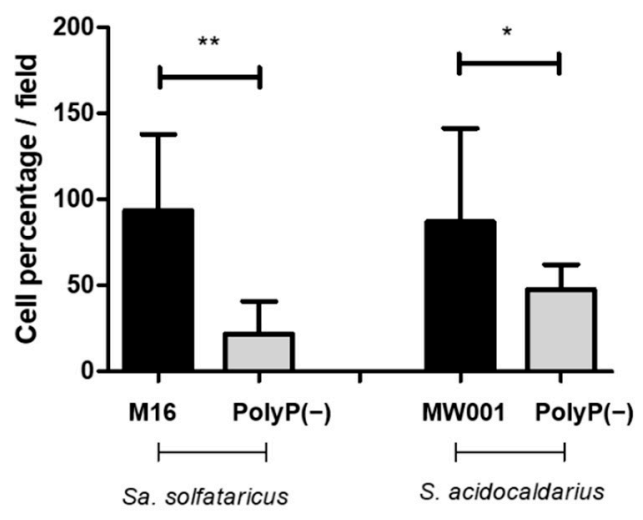

Figure 3. Effect of the lack of polyP in Sa. solfataricus and S. acidocaldarius cells attachment to a glass surface after $24 \mathrm{~h}$. (A) Microscopical image of MW001 and polyP (-) strains from S. acidocaldarius attached to glass slides. Cells were fixed with formaldehyde and stained with DAPI. Phase contrast $(\mathrm{PhC})$ and DAPI channels are shown. (B) Mean of the percentage of cell numbers per field. Each value was calculated in function of the average in the number of cells per field in the respective background strain (Sa. solfataricus M16 or S. acidocaldarius MW001). ANOVA test: ${ }^{*} p \leq 0.05$ and ${ }^{* *} p \leq 0.01$.

To further study other characteristics previously described for polyP (-) mutants in bacteria $[7,8]$, motility assays in semi solid gelrite plates were done. PolyP $(-)$ strains had only $30 \%$ swarming motility compared to the respective background strains (Figure 4). The observed phenomena-adhesion, motility, and biofilm formation-are all related to the mentioned surface structures in the cells, such as archaellum and adhesive pili [19,20]. Thus, polyP might be affecting levels, and/or the function of these structures. 
A

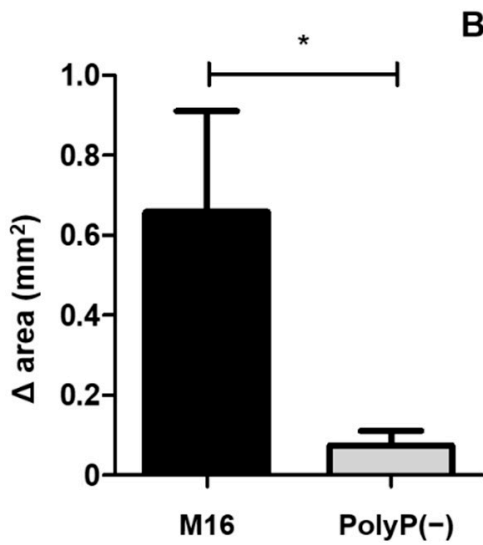

Sa. solfataricus
B

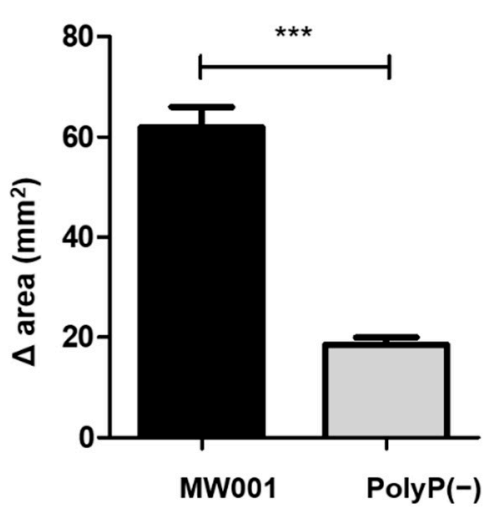

C

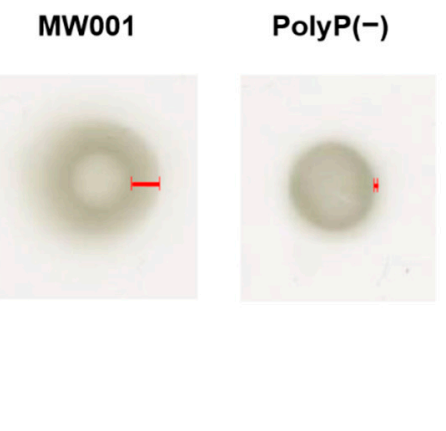

S. acidocaldarius

Figure 4. Motility assays of polyP (-) and WT strains of Sulfolobales. (A) Sa. solfataricus M16 and (B,C) S. acidocaldarius MW001. The results represent an average of at least 10 spots. ANOVA test: ${ }^{*} p \leq 0.05$ and ${ }^{* * *} p \leq 0.001$.

\subsection{Surface Structures Levels and Archaellum Assembly in PolyP (-) Mutants}

To find out whether the observed reduced motility of the polyP (-) strains was due to an altered expression of the components of the archaellum (the archaeal motility structure), the transcript levels of $a r l B$, encoding the structural component of the archaellum, were measured by qRT-PCR and compared between polyP (-) and background strains. In Sa. solfataricus, levels of arlB (SSO2323) did not show a significant difference (Figure 5A), whereas there was a higher arlB mRNA level in the polyP (-) strain of S. acidocaldarius (Saci_1178; Figure 5B). These results do not explain the reduced adhesion of the Sa. solfataricus polyP $(-)$, nor the reduced motility in both polyP $(-)$ strains (Figure $4 \mathrm{~A}$ ).

A.

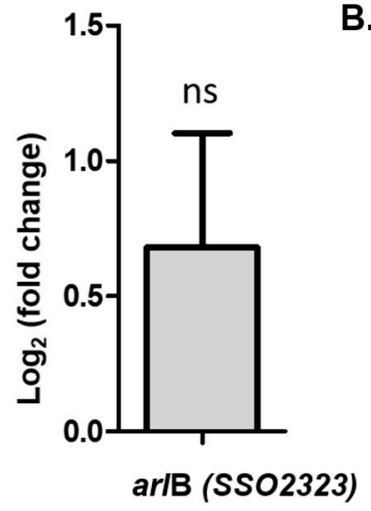

B.

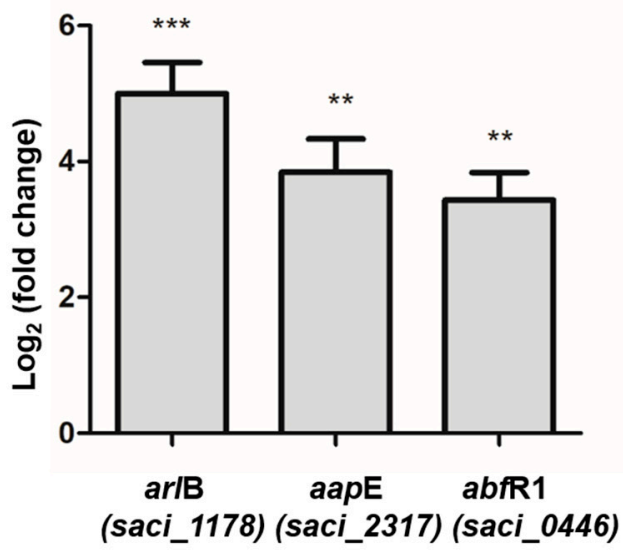

Figure 5. Relative gene expression of $\operatorname{arl} \mathrm{B}$, aap $E$ and $a b f R 1$ genes as measured by qRTPCR in polyP (-) versus WT in planktonic cells. (A) Sa. solfataricus (B) S. acidocaldarius. Wilcoxon Signed Rank Tests where ${ }^{* * *} p \leq 0.001,{ }^{* *} p \leq 0.01$ and ns: no significant.

The Aap-pilus is also involved in initial cell adhesion of S. acidocaldarius [19]. Thus, the levels of aapE mRNA, encoding for the ATPase energizing the assembly of this pilus were determined (Figure $5 \mathrm{~B}$ ). Almost 4 times higher levels of this component were found in polyP (-) cells compared to MW001.

Finally, the levels of abfR1 (Saci_0446) were measured. This gene codes for a protein that acts as a negative regulator of biofilm formation [10]. When phosphorylated AbfR1 does not inhibit biofilm formation and decreases its own expression [11], therefore it was of interest to test if the lack of polyP might affect the expression of this gene. As seen in 
Figure $5 \mathrm{~B}$, the expression levels of this gene were upregulated in the polyP (-) strain. This result could explain both the lower amount of biofilm formed by the polyP (-) strain in $S$. acidocaldarius, as well as the lower amounts of EPS seen by microscopy (Figure 2C,D).

\subsection{Archaellum Assembly in PolyP (-) Mutants}

It is not known how lack of polyP affects motility in cells, but it is possible that it is related to its role as an energy reservoir [4].

The results obtained here showed higher levels of the archaellin gene was being expressed in S. acidocaldarius polyP (-) strain. Besides the role of ATP in the archaellum rotation it is also known to play a function in the archaellum assembly [22]. Therefore, to determine whether the archaellum and other structures were being properly assembled on the cell surface, transmission electron microscopy (TEM) of S. acidocaldarius MW001 and polyP $(-)$ cells was done.

Archaellum formation can be induced by starvation [23]. S. acidocaldarius MW001 and polyP (-) strain subjected to starvation showed archaella as seen in S. acidocaldarius wild type strain (Figure 6A, red arrows) and in the control strain of S. acidocaldarius MW001-PPX (plasmid without induction) (Figure S3). In the polyP (-) strain, no archaellated cells could be perceived (Figure 6B). Pili could be seen in both strains, but in higher numbers in the WT strain (blue arrows).

A.

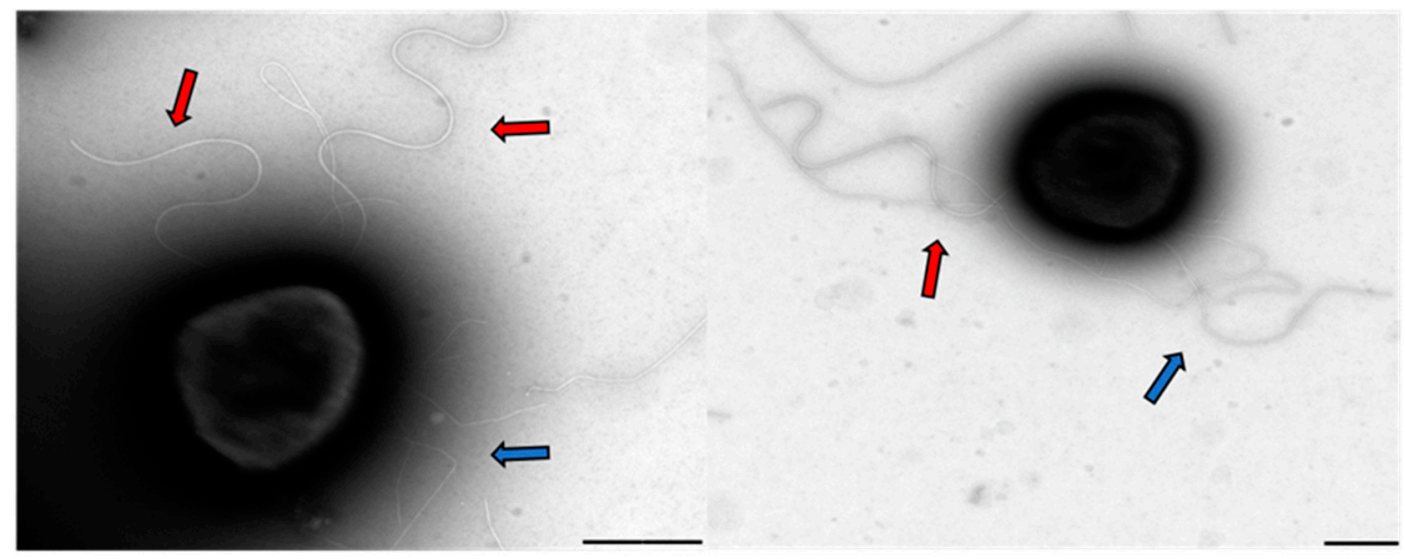

B.

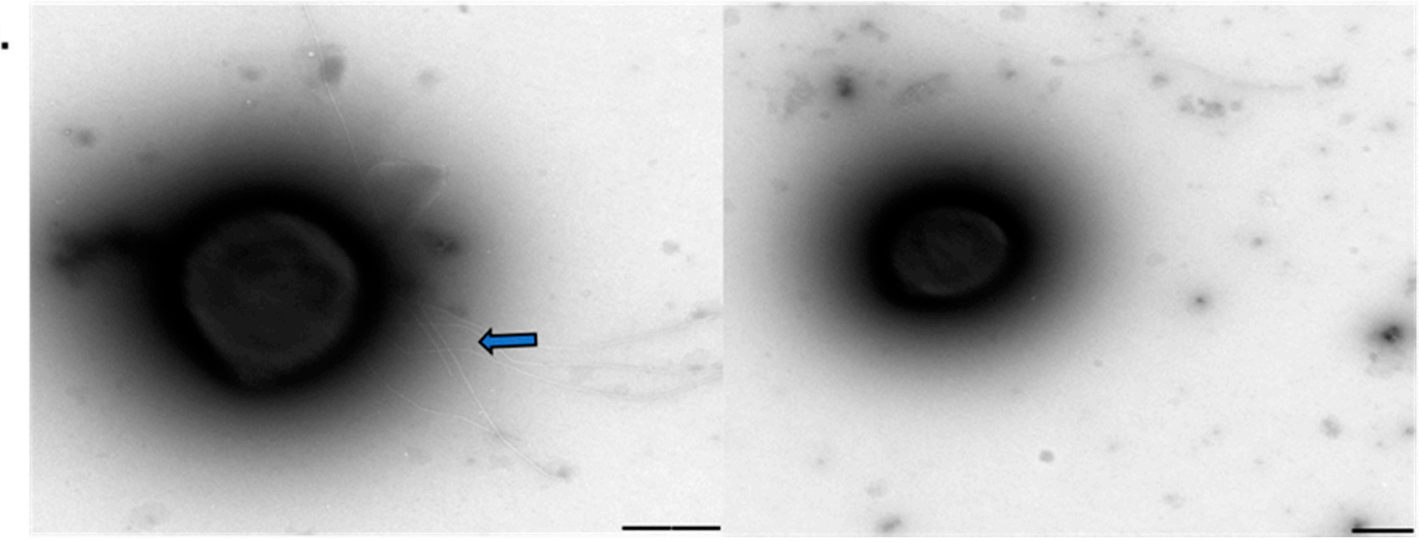

Figure 6. Transmission Electron Microscopy of S. acidocaldarius cells after starving conditions for archaellum induction. (A) MW001 (B) PolyP (-). Red arrows point to archaellum filaments. Blue arrows point to pili. The scale bars are $500 \mathrm{~nm}$.

This lack of archaellation in cells could explain the reduced biofilm formation and motility deficiency on semi solid gelrite plates (Figures 1 and 4). 


\section{Discussion}

Overexpression of the $p p x$ gene in both Sa. solfataricus and S. acidocaldarius eliminates polyP from their cells. Therefore, the use of these overexpression strains allowed to study the effect of polyP on biofilm formation, adhesion, and motility in archaeal cells.

Similar to what was observed for cells lacking the archaellum $[19,21]$, polyP $(-)$ cells exhibited a reduced surface-attachment and were not motile. Moreover, the S. acidocaldarius polyP (-) strain produced a compact biofilm, with less amounts of EPS as observed for the Aap-pilus deletion mutant and the double deletion mutant for archaellum and Aappilus [21]. The results presented here therefore suggest that similar to what is known in bacteria, polyP in Sulfolobales functions in biofilm formation and motility by regulating the production of cell-surface structures that are known to be involved in the initial cellular swimming and attachment to surfaces [19-21].

The exact mechanism of how polyP regulates biofilm formation is not known. In E. coli, the degradation of the polymer at the beginning of stationary phase is related to LuxS and the formation of biofilm [9]. In Sulfolobales, no Quorum sensing system is known. Therefore, polyP must regulate biofilm formation in a different way.

S. acidocaldarius contains two main regulators from the Lrs-14 family: Saci_1223 and AbfR1 [10]. AbfR1 was of particular interest since it is itself regulated by phosphorylation. AbfR1 acts by inhibiting biofilm formation via induction of the arl operon and reduction of EPS synthesis [11] (see Figure 7). AbfR1 also induces its own expression. However, when phosphorylated, AbfR1 cannot bind its regulation targets (arlB promoter and its own $a b f \mathrm{R} 1$ promoter regions). Here we found that $a b f \mathrm{R} 1 \mathrm{mRNA}$ levels are higher in the polyP (-) strain, which explains in part the lower amount of biofilm formation. A possible explanation to this phenomenon is that the lack of polyP affects the regulation of AbfR1 by impairing phosphorylation, which would lead not only to the over-expression of AbfR1 itself but also the overexpression of the arl operon (Figure 7).

Deficient protein phosphorylation-disturbing expression of the archaellum genes was seen before for S. acidocaldarius [24], where cells lacking protein phosphatases genes (Saci_0545 and Saci_0884) showed overexpression of archaellum but less motility on semisolid plates compared with the WT counterpart. However, Sa. solfataricus AbfR1 (SSO_0458) does not harbor the amino acids proposed to be phosphorylated in S. acidocaldarius (Y84 and S87) [11]. It is therefore unclear how polyP is affecting phosphorylation of this regulator in both species. It is possible that polyP might also function in providing energy for assembly of the archaellum and Aap-pili [22]. The assembly of the archaellum is energized by ATP hydrolysis [25]. In E. coli ppk and $p p x$ knockout mutants had lower expression of genes coding for fimbria and flagellum and were not flagellated as seen under the microscope [26]. Although Pseudomonas sp. B4 polyP (-) mutant showed lower levels of flagellin, intact flagella were also seen at its surface [8].

It is interesting to consider that $p p x$-gene overexpression could generate an artificial ATP-consuming futile cycle in the cells, which is expected to lower the availability of ATP and thus the general energy load of the microorganisms. In future studies, it will be of importance to consider this possible effect, especially since deprivation of ATP might also affect archaella assembly and its movements. In addition, measuring the effects on cellular ATP concentration or heat generation (by microcalorimetrically), or on the relative fitness of the cells, would also be of interest in potential studies.

It is possible that an eventual lack of energy might take place in both Sa. solfataricus and S. acidocaldarius strains that do not accumulate polyP due to the overexpression of their respective PPX enzymes. This might create a futile cycle due to the constant polyP degradation, which in turn can cause an eventual lack of energy in these Crenarchaeotes. This situation might in turn explain the lack of motility and low biofilm formation phenotypes reported in this work. It is known that both of these strains normally accumulate low levels of polyP in their cytoplasm [27]. Consequently, the amount of ATP required for the polymer synthesis should not be high. A bacterial kind of PPK type has not yet been identified in Sulfolobales. In addition, Sulfolobus species possess an ATP synthase 
enzymatic complex able to use the proton motive force to regenerate ATP [28]. Considering these species grow at acid $\mathrm{pH}$, ATP production could be favored in a natural way under their growth conditions. Each phosphate extension of the polyP polymer spends one ATP molecule and generates one of ADP. On the other hand, polyP degradation by the PPX enzyme would release one molecule of inorganic phosphate by each broken phosphodiester bond. Given these facts, one can consider that these archaeal strains could use both ADP and Pi substrates together with the energy of proton motive force to regenerate ATP from both polyP synthesis and degradation.

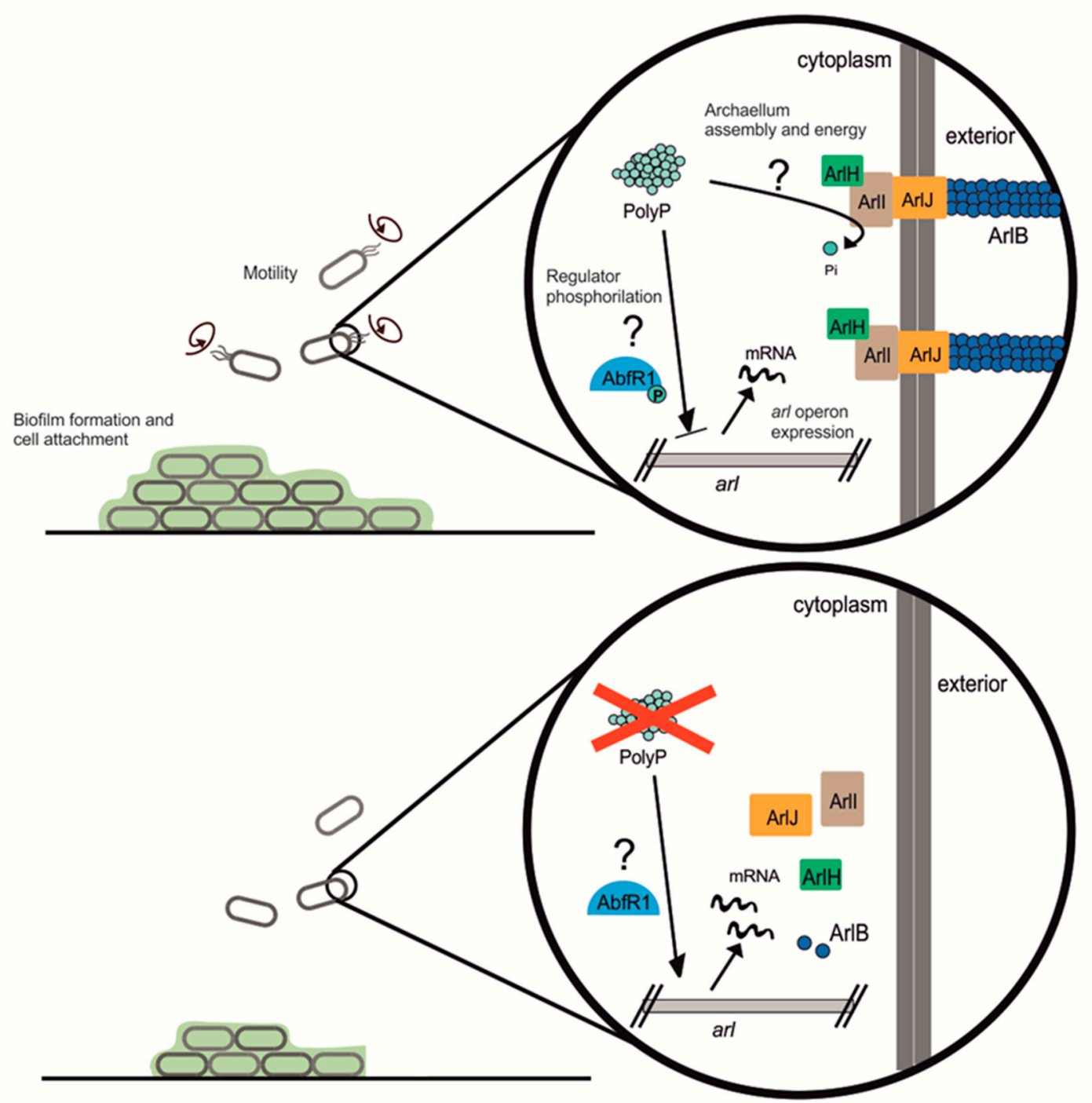

Figure 7. Cartoon showing the possible role of polyP in motility, adhesion, and biofilm formation in Sulfolobales.

\section{Conclusions}

Even though polyP plays many functions in both eukaryotic and prokaryotic species, only little is known about the role of polyP in archaea. Here we report that polyP is important in biofilm formation, adhesion, motility, expression, and assembly of the archaellum in crenarchaeotes $\mathrm{Sa}$. solfataricus and S. acidocaldarius. polyP (-) cells were not archaellated and showed less pili compared to the background strains used in this study. The lack of archaellum and pili in cell surface explains the lower motility and adhesion to glass surface, as well as some of the characteristics of the biofilm structure in these strains. We propose polyP might be affecting phosphorylation of proteins such as AbfR1, which is 
involved in biofilm formation as well as expression of Arl operon. At the same time, lack of polyP affects the assembly of the surface structures. Other levels of regulation should not be discarded meanwhile this study opens new questions about different roles of polyP in archaea.

This is the first evidence that polyP influences the mentioned phenomena in Archaea. By using genetically tractable archaea such as $S$. acidocaldarius, future studies would help us to determine the exact mode of regulation of archaeal polyP.

Supplementary Materials: The following are available online at https: / /www.mdpi.com/2076-2 607/9/1/193/s1, Figure S1: Lack of PolyP in S. acidocaldarius PolyP (-) strain, Figure S2: Growth curves comparing S. acidocaldarius MW001 and PolyP (-) strains, Figure S3: Representative TEM image from S. acidocaldarius MW001-PPX, Table S1: Strains used in this work, Table S2: Primers used in this work.

Author Contributions: Conceptualization, A.R., C.A.J., S.-V.A.; methodology, M.v.W., S.S., A.R.; investigation, M.v.W., S.S., A.R.; writing-review and editing A.R., M.v.W., S.S., S.-V.A., C.A.N., C.A.J. All authors have read and agreed to the published version of the manuscript.

Funding: This research was founded FONDECYT grants $\mathrm{N}^{\circ} 1150791$ and partially by $\mathrm{N}^{\circ} 1191554$ both to CAJ. AR doctoral studies and internship at Dr. Albers's Lab were fully funded by scholarship "Don Carlos Antonio Lopez" from the Government of Paraguay. The TEM is operated by the University of Freiburg, Faculty of Biology, as a partner unit within the Microscopy and Image Analysis Platform, Freiburg. AR would like to thank Lorena Saragoni from the Microscopy facilities (Centro de Equipamiento Mayor) of the University of Chile. SS was funded by SFB 1381 (Deutsche Forschungsgemeinschaft (German Research Foundation) under project no. 403222702-SFB 1381). MvW received support from the VW foundation grant "Momentum" (VW Stiftung grant number 94933).

Institutional Review Board Statement: Not applicable.

Informed Consent Statement: Not applicable.

Data Availability Statement: The data presented in this study are openly available.

Conflicts of Interest: The authors declare no conflict of interest.

\section{References}

1. Kornberg, A.; Rao, N.N.; Ault-Riché, D. Inorganic polyphosphate: A molecule of many functions. Annu. Rev. Biochem. 1999, 68, 89-125. [CrossRef] [PubMed]

2. Cardona, S.T.; Chavez, F.P.; Jerez, C.A. The exopolyphosphatase gene from Sulfolobus solfataricus: Characterization of the first gene found to be involved in polyphosphate metabolism in Archaea. Appl. Environ. Microbiol. 2002, 68, 4812-4819. [CrossRef] [PubMed]

3. Wang, L.; Liu, Q.; Wu, X.; Huang, Y.; Wise, M.J.; Liu, Z.; Wang, W.; Hu, J.; Wang, C. Bioinformatics analysis of metabolism pathways of archaeal energy reserves. Sci. Rep. 2019, 9, 1-12. [CrossRef] [PubMed]

4. Albi, T.; Serrano, A. Inorganic polyphosphate in the microbial world. Emerging roles for a multifaceted biopolymer. World J. Microbiol. Biotechnol. 2016, 32, 1-12. [CrossRef]

5. Drozd, M.; Chandrashekhar, K.; Rajashekara, G. Polyphosphate-mediated modulation of Campylobacter jejuni biofilm growth and stability. Virulence 2014, 5, 680-690. [CrossRef]

6. $\quad$ Rashid, M.H.; Rumbaugh, K.; Passador, L.; Davies, D.G.; Hamood, N.; Iglewski, B.H.; Kornberg, A. Polyphosphate kinase is essential for biofilm development, quorum sensing, and virulence of Pseudomonas aeruginosa. Proc. Natl. Acad. Sci. USA 2000, 97, 9636-9641. [CrossRef]

7. Shi, X.; Rao, N.N.; Kornberg, A. Inorganic polyphosphate in Bacillus cereus: Motility, biofilm formation, and sporulation. Proc. Natl. Acad. Sci. USA 2004, 101, 17061-17065. [CrossRef]

8. Varela, C.; Mauriaca, C.; Paradela, A.; Albar, J.P.; Jerez, C.A.; Chávez, F.P. New structural and functional defects in polyphosphate deficient bacteria: A cellular and proteomic study. BMC Microbiol. 2010, 10, 7. [CrossRef]

9. Grillo-Puertas, M.; Rintoul, R.; Villegas, J.M.; Rapisarda, V.A. Polyphosphate degradation in stationary phase triggers biofilm formation via LuxS quorum sensing system in Escherichia coli. PLoS ONE 2012, 7, e50368. [CrossRef]

10. Orell, A.; Peeters, E.; Vassen, V.; Jachlewski, S.; Schalles, S.; Siebers, B.; Albers, S.V. Lrs14 transcriptional regulators influence biofilm formation and cell motility of Crenarchaea. ISME J. 2013, 768, 1886-1898. [CrossRef]

11. Li, L.; Banerjee, A.; Bischof, L.F.; Maklad, H.R.; Hoffmann, L.; Henche, A.L.; Veliz, F.; Bildl, W.; Schulte, U.; Orell, A.; et al. Wing phosphorylation is a major functional determinant of the Lrs14-type biofilm and motility regulator AbfR1 in Sulfolobus acidocaldarius. Mol. Microbiol. 2017, 105, 777-793. [CrossRef] 
12. Soto, D.F.; Recalde, A.; Orell, A.; Albers, S.V.; Paradela, A.; Navarro, C.A.; Jerez, C.A. Global effect of the lack of inorganic polyphosphate in the extremophilic archaeon Sulfolobus solfataricus: A proteomic approach. J. Proteom. 2019, 191, 143-152. [CrossRef] [PubMed]

13. Van der Kolk, N.; Wagner, A.; Wagner, M.; Waßmer, B.; Siebers, B.; Albers, S.V. Identification of XylR, the activator of arabinose/xylose inducible regulon in Sulfolobus acidocaldarius and its application for homologous protein expression. Front. Microbiol. 2020, 11, 1-9. [CrossRef] [PubMed]

14. Wagner, M.; Van Wolferen, M.; Wagner, A.; Lassak, K.; Meyer, B.H.; Reimann, J.; Albers, S. Versatile genetic toolbox for the crenarchaeote Sulfolobus acidocaldarius. Front. Microbiol. 2012, 3, 1-12. [CrossRef] [PubMed]

15. Koerdt, A.; Gödeke, J.; Berger, J.; Thormann, K.M.; Albers, S.V. Crenarchaeal biofilm formation under extreme conditions. PLoS ONE 2010, 5, e14104. [CrossRef] [PubMed]

16. Schindelin, J.; Arganda-Carreras, I.; Frise, E.; Kaynig, V.; Longair, M.; Pietzsch, T.; Preibisch, S.; Rueden, C.; Saalfeld, S.; Schmid, B.; et al. Fiji: An open-source platform for biological-image analysis. Nat. Methods 2012, 9, 676-682. [CrossRef]

17. Orell, A.; Remonsellez, F.; Arancibia, R.; Jerez, C.A. Molecular characterization of copper and cadmium resistance determinants in the biomining thermoacidophilic archaeon Sulfolobus metallicus. Archaea 2013, 2013, 289236. [CrossRef]

18. Martusewitsch, E.; Sensen, C.W.; Schleper, C. High spontaneous mutation rate in the hyperthermophilic archaeon Sulfolobus solfataricus is mediated by transposable elements. J. Bacteriol. 2000, 182, 2574-2581. [CrossRef]

19. Zolghadr, B.; Kling, A.; Koerdt, A.; Driessen, A.J.M.; Rachel, R.; Albers, S.V. Appendage-mediated surface adherence of Sulfolobus solfataricus. J. Bacteriol. 2010, 192, 104-110. [CrossRef]

20. Van Wolferen, M.; Orell, A.; Albers, S.V. Archaeal biofilm formation. Nat. Rev. Microbiol. 2018, 16, 699-713. [CrossRef]

21. Henche, A.L.; Koerdt, A.; Gosh, A.; Albers, S.V. Influence of cell surface structures on crenarchaeal biofilm formation using a thermostable green fluorescent protein. Environ. Microbiol. 2012, 14, 779-793. [CrossRef] [PubMed]

22. Albers, S.V.; Jarrell, K.F. The Archaellum: An update on the unique archaeal motility structure. Trends Microbiol. 2018, 26, 351-362. [CrossRef] [PubMed]

23. Lassak, K.; Ghosh, A.; Albers, S.-V. Diversity, assembly and regulation of archaeal type IV pili-like and non-type-IV pili-like surface structures. Res. Microbiol. 2012, 163, 630-644. [CrossRef] [PubMed]

24. Reimann, J.; Esser, D.; Orell, A.; Amman, F.; Pham, T.K.; Noirel, J.; Lindås, A.-C.; Bernander, R.; Wright, P.C.; Siebers, B.; et al. Archaeal signal transduction: Impact of protein phosphatase deletions on cell size, motility, and energy metabolism in Sulfolobus acidocaldarius. Mol. Cell. Proteom. 2013, 12, 3908-3923. [CrossRef] [PubMed]

25. Hoffmann, L.; Schummer, A.; Reimann, J.; Haurat, M.F.; Wilson, A.J.; Beeby, M.; Warscheid, B.; Albers, S.V. Expanding the archaellum regulatory network-the eukaryotic protein kinases ArnC and ArnD influence motility of Sulfolobus acidocaldarius. Microbiologyopen 2017, 6, e00414. [CrossRef] [PubMed]

26. Varas, M.; Valdivieso, C.; Mauriaca, C.; Ortíz-Severín, J.; Paradela, A.; Poblete-Castro, I.; Cabrera, R.; Chávez, F.P. Multi-level evaluation of Escherichia coli polyphosphate related mutants using global transcriptomic, proteomic and phenomic analyses. Biochim. Biophys. Acta-Gen. Subj. 2017, 1861, 871-883. [CrossRef]

27. Orell, A.; Navarro, C.A.; Rivero, M.; Aguilar, J.S.; Jerez, C.A. Inorganic polyphosphates in extremophiles and their possible functions. Extremophiles 2012, 16, 573-583. [CrossRef]

28. Hochstein, L.I.; Stan-Lotter, H. Purification and Properties of an ATPase from Sulfolobus solfataricus. Arch. Biochem. Biophys. 1992, 295, 153-160. [CrossRef] 